\title{
Using a Swarm of Self-propelled Natural Microrobots in the Form of Flagellated Bacteria to Perform Complex Micro-assembly Tasks
}

\author{
Sylvain Martel, Senior Member, IEEE, and Mahmood Mohammadi
}

\begin{abstract}
Many science fiction novels have envisioned swarms of artificial microrobots capable of performing complex collective tasks. Unfortunately, today's technological constraints have prevented such powerful concept to be a reality when considering artificial microrobots. In this paper, we show that a swarm of computer-controlled flagellated Magnetotactic Bacteria (MTB) acting as natural microrobots of approximately 1 to 2 micrometers in diameter can perform many of the same complex collective tasks envisioned with these futuristic self-propelled artificial microrobots. To prove the concept, magnetotaxis-based control has been used to coordinate a swarm made of thousands of these self-propelled natural microrobots to build in a collective effort, a miniature version of an ancient Egyptian pyramid.
\end{abstract}

\section{INTRODUCTION}

$\mathrm{B}$ ACTERIA can be used as computer-controlled bioactuators and means of propulsion with accurate steering for microrobots and other micro-scaled entities to accomplish precise operations as suggested in [1] and demonstrated experimentally in [2]. The last reference confirmed that the propulsion force provided by the flagella being connected to molecular motors embedded in the bacterial cell and the chain of nanoparticles referred to as magnetosomes acting as a miniature steering mechanism under the control of an external computer, can be exploited to replace current technologies being presently used in macro-scale robotics but which could not be implemented at the micro-scale due to technological constraints. This strategy relies on harnessing instead of mimicking nature to compensate for unavailable technologies, by using flagellated bacteria and more specifically Magnetotactic Bacteria (MTB) for not only propulsion and transport, but also for the controlled steering or computerized directional swimming control of bacterial micro- nanorobots [3], i.e. micro- nanorobots being propelled by bacteria. The latter aimed at embedding natural with artificial or synthetic

Manuscript received September 15, 2009. This work was supported in part by the Canada Research Chair (CRC) in Micro/Nanosystem Development, Fabrication and Validation and grants from the National Sciences and Engineering Research Council of Canada (NSERC), the Province of Québec, and the Canada Foundation for Innovation (CFI).

S. Martel (corresponding author) is with the NanoRobotics Laboratory, Department of Computer and Software Engineering, and the Institute of Biomedical Engineering, École Polytechnique de Montréal (EPM), Montréal (Québec), P.O. Box 6079 Station Centre-ville, H3C 3A7 Canada (phone: 514-340-4711 ext. 5098; fax: 514-340-4658; e-mail: sylvain.martel@polymtl.ca).

M. Mohammadi is also with the NanoRobotics Laboratory at École Polytechnique de Montréal (EPM). components to construct what we refer to as hybrid microrobots.

Connecting artificial or synthetic components to a natural self-propelled entity that can be controlled by computer is a very powerful concept with many potential applications. For instance, antibodies can be used to attach functionalized polymeric nanoparticles containing therapeutic agents to the cell of a flagellated bacterium. A swarm of such hybrid microrobots operating under computer control can then be used for targeting tumoral lesions in the human body for the delivery of therapeutic agents [4]. It was shown in [5] that the flagellated molecular motors embedded in each MC-1 bacterium being propelled by two flagella bundles providing a thrust force exceeding $4 \mathrm{pN}$, were more suitable than any other technologies when operating in the microvasculature while being trackable in the human body using Magnetic Resonance Imaging (MRI).

Extending this idea of attaching particles to flagellated bacteria, accurate manipulation of micro-scaled objects can also be considered as demonstrated experimentally in [6]. In the early stage, the object being manipulated or transported precisely to specific pre-determined locations was a 3micrometer $(\mu \mathrm{m})$ bead being attached to a flagellated bacterial cell (diameter between $\sim 1-2 \mu \mathrm{m}$ ) being guided and controlled using computer software. Although we showed that slightly larger micro-beads can also been manipulated in this manner as depicted in Fig. 1, more recently, we have demonstrated in [7] the possibility of using a swarm of flagellated bacteria to move or propel much larger untethered micro-objects with dimensions ranging from tens to several hundreds of micrometers, the latter providing propulsion force sufficient for larger entities such as bacterial intelligent microrobots relying on embedded microelectronics to communicate and to accomplish specific tasks.

Nonetheless, although we have achieved accurate computer-controlled propulsion and transport, including the manipulation of single micro-objects along pre-programmed paths using flagellated bacteria, no micro-assembly relying on the coordinated transport and manipulation of several micro-components have been done. This new type of microassembly that could be referred to as bacterial microassembly could offer new opportunities in the field of microrobotics as well as for the implementation of bacterial microfactories. 


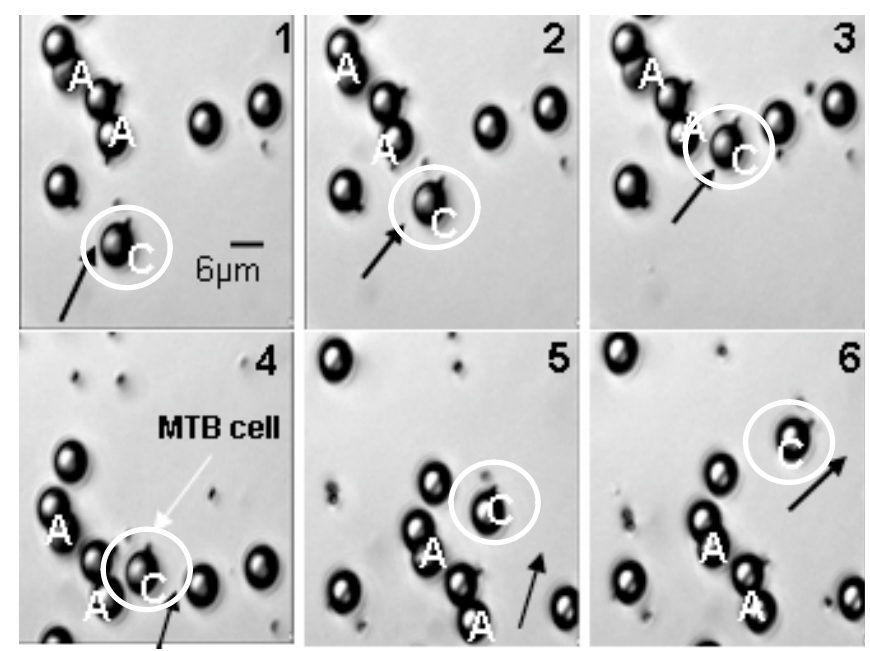

Fig. 1. One among many examples recorded in our research laboratory showing a bead being transported by a single flagellated bacterium and acting under computer control. Here the bead identified in the photographs as $\mathrm{C}$ had a diameter of 6.2 micrometers and was transported by a single MC-1 MTB cell. The smaller dots in the photographs are other MC-1 flagellated bacteria not attached to bead. The two beads identified A did not have any bacteria attached to and are shown here as references to show the motion of the transported bead $\mathrm{C}$.

The general concept of a bacterial micro-factory is similar to its macro-scale counterpart which relies on a given number of human workers which can scale according to the need of a given assembly task. For the micro-factory, each human worker is replaced by a bacterium. Similarly, as in a human scale factory involved in complex tasks, the group of workers will typically be coordinated by a supervisor, such as an external computer in our particular case. Although previous experiments were done with bacteria being attached to the objects being manipulated, here micro-manipulation of a single component to be assembled is being performed without attaching the bacteria to the object itself. This allows the same micro-workers to be dispatched to the transport or manipulation of several micro-components as well as other assembly tasks. Such new concept is demonstrated and explained further throughout the following sections.

\section{MAIN COMPONENTS USED FOR THE PROOF-OF-CONCEPT}

\section{A. Selected Micro-structure}

As for the ancient man-made structures which relied on the collecting force of a sufficient number of workers to move each large and heavy block to be assembled, the approach proposed here also relies on collecting the required force from a sufficient number of micro-workers represented by flagellated bacteria to move each block or microcomponent to be assembled. Since this is a first proof-ofconcept, the Step pyramid being among the first structures being built by humans has been selected here to show the potential of this method.

The Step pyramid was the earliest form of pyramid dating back to the Third Dynasty. The first and probably the only Step pyramid ever completed, is that of King Netjerykhet Djoser at Saqqara. This Step pyramid known as the Djoser pyramid is shown in Fig. 2 and was a predecessor to the development of the True pyramids which have smoother edges. The Step pyramid is certainly not near as pleasing to the eye as the True pyramid but it represents a symbol of the beginning of the construction of many more man-made structures of increasing sophistication. Similarly, the Step pyramid chosen as the first structure being build by the bacteria is certainly not the best looking structure but as history proved it, it may as it happened for humans, certainly suggest that more sophisticated bacteria-made structures could soon be constructed.

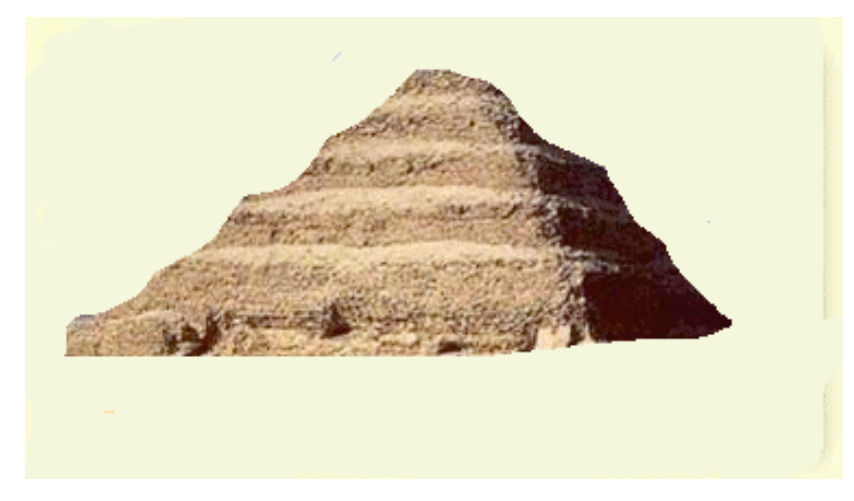

Fig. 2. Photograph of the Djoser Step pyramid known as an important, initial milestone in the history of man-made structures. This pyramid has inspired the new micro-assembly method proposed in this paper where thousands of human workers of the ancient Egypt have been replaced by thousands of micro-workers represented by flagellated magnetotactic bacteria of type MC-1, each having a diameter of approximately 2 micrometers.

\section{B. Selected Micro-workers}

The fact that what could be achieved in term of building structures by using humans as workers could also be repeated in some ways when using bacteria but at a smaller scale could potentially certainly prove to be true especially if modern man-made technologies and micro-robotic technologies in particular, could be integrated adequately to exploit the efficiency of flagellated bacteria to perform work at the micro-scale. Indeed, presently the MC-1 bacteria selected as our micro-workers and cultivated in our research laboratory under specific conditions can move in an aqueous environment at a velocity exceeding 100 to 150 times its own body (cell) length, i.e. 200 to $300 \mu \mathrm{m} / \mathrm{s}$. This is extremely fast compared to other species of flagellated bacteria. For instance, the well known Escherichia Coli swims at speeds of about $30 \mu \mathrm{m} / \mathrm{s}$. Since the thrust force propels the bacteria forward, a higher swimming speed means a higher thrust force which as discussed later, will prove to be a great advantage during the micro-manipulation of micro-objects. Furthermore, our previous experiments also showed that they can maintain this cadence for several hours when working in proper conditions. 


\section{Building Blocks}

The building blocks to be assembled as a Step pyramid were made of SU-8 and developed using standard photolithography procedures and transparency mask. Although different sizes could have been used, the building blocks had arbitrary chosen dimensions of $80 \mu \mathrm{m} \times 20 \mu \mathrm{m} \times$ $15 \mu \mathrm{m}$ (length $\times$ width $\times$ thickness). These values were chosen with a length corresponding approximately to the human hair thickness while showing that the method can manipulate objects with overall dimensions greater that what would have been possible with other known micromanipulation techniques such as optical tweezers, to name but only one classical technique.

\section{GENERAL METHOD}

A mechanical-based micro-manipulation approach compared to other micro-manipulation methods in a fluid environment such as optical tweezers, electrophoresis, etc., has many advantages including but not limited to the fact that it is independent of the material properties (e.g. dielectric properties) of the object being manipulated. But typically, unlike other approaches mentioned above, mechanical-based micro-manipulations and assemblies make direct contacts with the objects which may not always be desirable or practical (e.g. when in an enclosure or sealed environment). A possible alternative could be the use of directional flows generated from pumping fluid to move a specific micro-object to a desired direction and location. Unfortunately, the number of micro-pumps and/or pumping channels required to accomplish accurate micromanipulations could be quite significant. Furthermore, since the source of the flow used to move an object can be relatively far from the object being manipulated, in many cases other neighbored objects will likely be influenced by such a flow and move out of their intended position. Such issue will most likely prevent accurate micro-assemblies to be performed.

One potential solution would be to dispatch a sufficient number of miniature pumps and orient them properly in the vicinity and close enough to the micro-object being manipulated in such a manner that the generated flow would not interfere significantly with other neighbored components. Such pump may take the form of small propellers such as the flagellated motors of bacteria. When combined with pushing action by other bacteria in the swarm, a powerful yet flexible micro-manipulation method can be implemented.

Flagellated bacteria use rotary motors at the cell wall to rotate their helical flagella. Since this motion always pushes fluid along the same axial direction for a given sense of rotation, the motion is not reciprocal and generates net thrust. Such principle of pumping fluid from the flagellated bacteria has been demonstrated experimentally [10]. In the latter, a dense layer of flagellated bacteria were attached at an angle to a microfluidic channel wall to pump fluid. This so called bacterial carpet was fixed and as such, it would have the similar limitations as the ones mentioned previously to accomplish accurate micro-assemblies. Nonetheless, the results showed that flagellated bacteria can be used as micro-pumps.

Another important fact in the point of view of microassembly is that the bacteria are working in a very low Reynolds environment. Laminar flow occurs in such an environment where viscous forces are dominant, and is characterized by smooth, constant fluid motion. Such environment does not have the turbulent flow that occurs at high Reynolds numbers where inertial forces dominate, which tend to produce random eddies, vortices and other flow instabilities which may contribute in imprecision during manipulation in a fluid medium. Hence, in a low Reynolds environment characterized with extremely low inertia that can be considered negligible in our application, the micro-object being manipulated will instantly start or stop with the corresponding flow or pushing action generated from the bacteria.

Several stimuli may affect the directional motion of flagellated bacteria. Chemical gradients, oxygen and light are typical examples. Chemotaxis-based bacteria reverse their rotary motor for changing direction in a random manner. Indeed, a unique feature of flagella motors in wildtype cells is that they alternate clockwise and counterclockwise rotation in a random manner. Such randomness is not suitable when involved in precise microassembly tasks. Furthermore, studies have shown that when the bacteria move forward without any change in the direction of rotation of the molecular motors, that significant deviations from an ideal straight line still occur.

In order to avoid such randomness as well as guaranteeing a straight line of motion when desired, the flagellated bacteria are dispatched towards the micro-object to be manipulated using magnetotaxis [8]. As such, MTB seem to be the ideal candidates for such applications while supporting direct computer control. Such control was performed by a coils configuration capable of generating lines of magnetic field in any desired directions. Such directional field induced a torque on the chain of magnetosomes in each cell the same manner as the direction of a compass needle is being influenced by the magnetic field.

\section{A. Swarming}

Using this principle of magnetotaxis-based directional control while maintaining the magnetic field intensity at a few Gauss can achieve a better response of each MTB while making negligible other sensory means (e.g. chemotaxis, aerotaxis, and phototaxis) of the bacteria which are not under computer control and which could yield displacement error. Using this method, MC-1 bacteria initially spread in a liquid medium can be directed to form a swarm using a special non-uniform field forcing them to concentrate at a specific location. This is depicted in Fig. 3. As depicted in Fig. 3A, it will typically take a few seconds to a few minutes to assemble a swarm or aggregate of up to many thousands 
of these micro-workers depending on the required number of flagellated bacteria to accomplish the task. This will depend on the characteristics such as the size of the building blocks that needed to be transported and assembled. Also as depicted in Fig. 3B and measured experimentally, each MC1 bacterium can contribute to an increase of the overall thrust force of the swarm with an increment between 4.0 to $4.7 \mathrm{pN}$ being generated by two flagella bundles per cell.

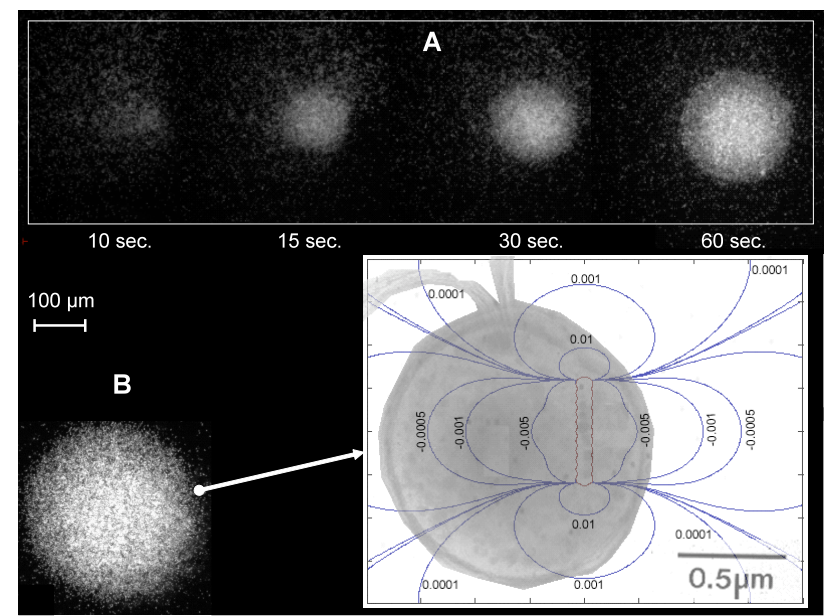

Fig. 3. Swarm or aggregate of several thousand MC-1 flagellated bacteria. A. Example of a formation of a swarm as seen under an optical microscope. The sequences taken in the first minute provide an idea of the time required to assemble several thousand micro-workers needed to transport relatively large blocks to be assembled in order to build a coherent structure. B. Image of a larger swarm where each dot in the swarm is a micro-worker represented by the MC-1 bacterium shown on the right. The two flagella bundles used for propulsion and the chain of magnetosomes used for directional control are easily visible on the photograph of the bacterium.

\section{B. Bacterial Micro-assembly}

Although we have showed that this proved to be sufficient to move objects with diameter up to approximately $6.2 \mu \mathrm{m}$ as depicted in Fig. 1, this force can be scaled up by adding more thrust force with more micro-workers. Furthermore, the advantages of a collective force assembled with a swarm as shown in Fig. 3, is that the same population of microworkers or bacteria can be used many times to transport different blocks during a relatively long period of time without the need to inject additional bacteria.

The swimming of each bacterium above an infinite plane rigid boundary is treated as a so-called "outer flow problem" in low Reynolds number fluid dynamics. Because of the small overall size of the bacterium $(1-2 \mu \mathrm{m})$ and the Reynolds number of the associated fluid motion $\left(\sim 10^{-5}\right)$, the fluid motion is governed by the Stokes equation:

$$
\nabla p(x)=\mu \nabla^{2} u(x), \quad \nabla \cdot u(x)=0 .
$$

where $p, \mu, u$, and $x$ denote the pressure, the viscosity, the velocity vector, and the position vector, respectively. Since each bacterium is self-propelled, i.e. that it swims without the use of an external force, the free-swimming condition applies:

$$
\int_{S} t(x) d S=0 \quad \text { for net force, }
$$

$$
\int_{S} r \times t(x) d S=0 \text { for net torque, }
$$

where $t$ is the traction vector referring to the surface force per unit area and $r$ is the position vector with an arbitrary origin. The thrust force provided by each MTB is one of the key factors to consider in this application. Such thrust force can be estimated from the terminal velocity $v_{T}$ of the $\mathrm{MC}-1$ MTB which were measured in a free medium. From Stokes' equation:

$$
F_{T}=\frac{v_{T}}{3 \pi \eta \quad d},
$$

The thrust force is estimated at $4.7 \times 10^{-12} \geq F_{T} \geq 4.0 \times 10^{-12} \mathrm{~N}$ with $\eta=1.0 \mathrm{mPa} \cdot \mathrm{s}$ in water at $20^{\circ} \mathrm{C}$ being the viscosity of the medium, while $d \approx 2 \times 10^{-6} \mathrm{~m}$ represents the diameter of the cell when unloaded. For moving an object in a liquid medium, the method can be divided into two major force components being the effective horizontal force and the effective vertical force being designated $F_{H}$ (pushing action by MTB) and $F_{V}$ (generated by the bacterial carpet) respectively. The horizontal force component is responsible for moving the object while the vertical force component is used to reduce or eliminate the interacting force or forces between the substrate and the component to be moved and designated here $F_{N}$ that may act against the horizontal force component, preventing in many cases the displacement of such object. To be able to move an object, the following condition must then be satisfied within the conditions expressed in Eq. 1, 2 and 3:

$$
F_{H}>D_{C}, \quad F_{V}>F_{N} .
$$

where $D_{C}$ is the drag force of the object being manipulated. The horizontal force $F_{H}$ should be larger with a larger exposed surface used to push the micro-object if there are enough bacteria to cover such surface. To eliminate (or reduce) $F_{N}$, the object must be lifted from the substrate. This is achieved by implementing a bacterial carpet between the substrate and the object or component to be displaced. A roughness of only a few micrometers between the component and the surface is sufficient to allow the flagellated bacteria to be positioned as to form a bacterial carpet under the object. This is done by orienting and concentrating the lines of magnetic field in the center of the horizontal plane of the micro-object in such a way as to orient the flagella away from the substrate and hence, towards the object itself. With sufficient horizontal surface, the flow generated by the micro-propellers provided by the bacterial carpet can be sufficient to lift the object (or for some geometries, to provide a layer between the two interacting surfaces to ease the displacement) and to allow other bacteria in the swarm to move such object in the desired location. The lines of magnetic field are then shifted along a planned trajectory to move the swarm and hence $F_{H}$ and $F_{V}$ simultaneously. 


\section{EXPERIMENTAL RESULTS}

An example of bacterial micro-assembly is depicted in Fig. 4 and Fig. 5 showing approximately 5000 bacteria being involved in the construction of a miniature Step pyramid. The bacteria acting under computer coordination were working in an aqueous medium (PBS 1X Tween 80, 0.05\%) at room temperature. The whole assembly process took $\sim 15$ min. and was recorded using a Zeiss AxioImager Z1 optical microscope with a Sony camera.
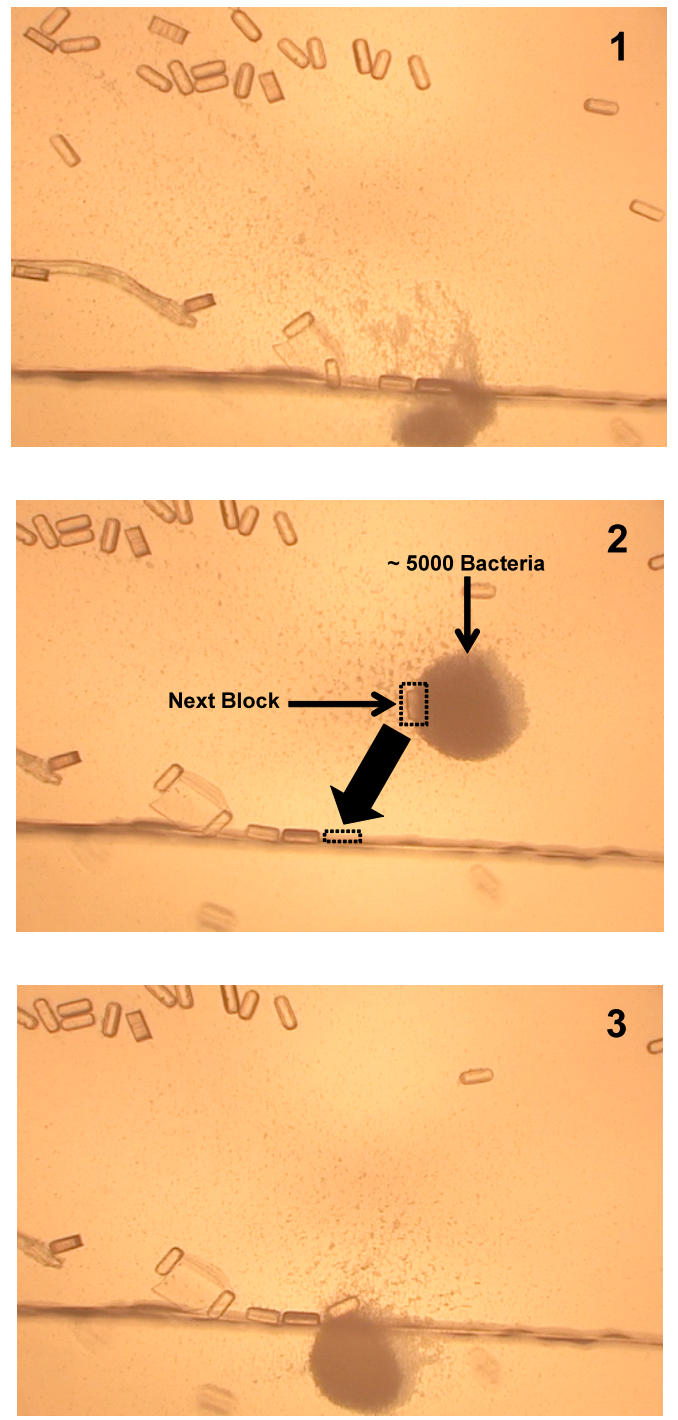

Fig. 4. Images taken under an optical microscope showing the initial microassembly process performed by the bacteria to construct the base of the Step pyramid. In the first image, the second block of the base of the pyramid has been placed next to the first block. In the second image, another block (surrounded by a dotted square and indicated by an arrow) to complete the base of the pyramid is being transported by the aggregate of approximately 5000 flagellated magnetotactic bacteria of type MC-1. The thick arrow in the second image points to the planned location where the last block of the base of the pyramid must be placed and retained by the edge created by a cover glass placed the aqueous medium. In the third image, the base of the Step pyramid is almost completed. In the same image, the last block is being placed and adjusted to be positioned in the right location.

The images depicted in Fig. 4 and in Fig. 5 show the aggregate of approximately $5000 \mathrm{MC}-1$ bacteria being controlled to transport and assemble blocks to build a Step pyramid somewhat similar to the one shown in Fig. 2. In this example, the pyramidal structure was built one block at a time and took approximately 15 minutes to complete. These images show not only the first structure being constructed by bacteria, but it also prove that the force provided by flagellated bacterial cells can be exploited to accomplish relatively complex micro-manipulation and assembly tasks.
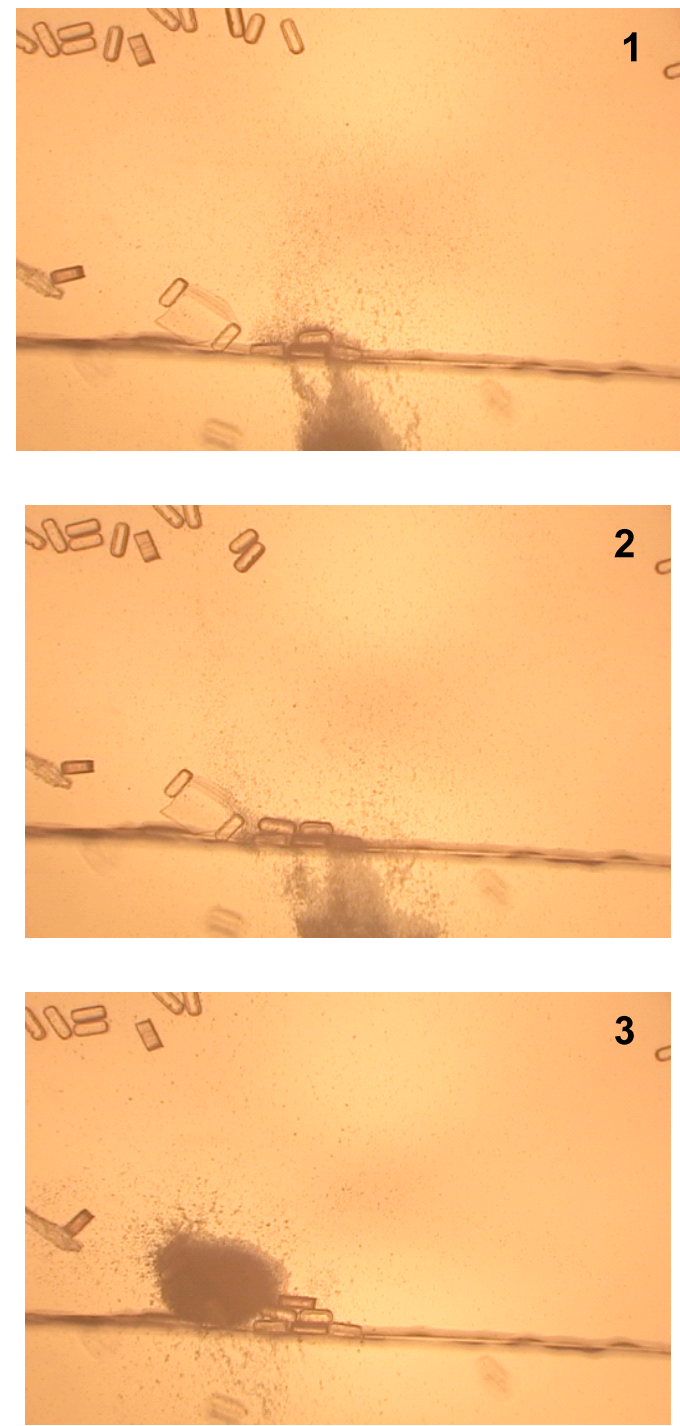

Fig. 5. Completion of the first Step pyramid built with bacteria. In the first image, the first block of the second level is placed on top of the base. In the second image, the second level is completed with the placement of another block. Finally in the last image, the Step pyramid is completed. In the last image, the size of the aggregate of approximately 5000 bacteria is seen with respect to the size of the pyramid.

The final Step pyramid shown in Fig. 6 and which was built initially with only 6 blocks may not look as good as the human-made Step pyramid depicted in Fig. 2, but unlike the latter which took many years to complete, the one depicted in Fig. 6 only took approximately 15 minutes using blocks with overall sizes and weight with were much more difficult 
to work when compared with the size and weight of each individual workers.

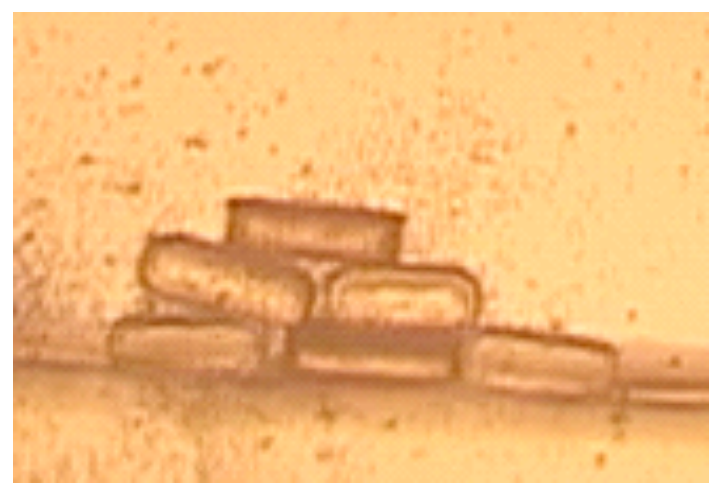

Fig. 6. The final Step pyramid. This is a first-proof-of-concept proving the feasibility of using bacteria for micro-manipulation and micro-assembly.

\section{DiscusSION}

Micromanipulation is known to be the robotic manipulation of objects with characteristic dimensions in the micrometer range. When several of such objects are manipulated in a coherent and coordinated manner, then larger structures can be built through micro-assembly [9].

In order to increase the throughput rate of micro-assembly while decreasing the associated cost, a scalable approach where parallel assembly processes can be performed while avoiding the hardware complexity required in many existing methods, is needed. Actual micro-manipulation and microassembly approaches can be categorized as electrical and magnetic (e.g. dielectrophoresis, etc.), mechanical (e.g. STM, AFM, mechanical micro-grippers and tweezers, etc.), and optical (e.g. optical tweezers or optical trapping), and others such as acoustic forces and direct self-assembly relying on components and interactions that are tailored to form a desired structure. The assembly process of the latter would typically rely on electrostatic interactions, mechanical vibrations, and/or surface tensions to name but a few examples. The new micro-manipulation and micro-assembly approach briefly presented here has many advantages that the above-mentioned approaches do not have. First, the approach can be highly scalable depending on the geometry of the micro-objects being manipulated and can provide force beyond what can be provided by technique such as the optical tweezers and without the complexity and cost. Bacterial micro-assembly as described here is not only very low cost while required very low electrical power since it relies mostly on the motility of bacteria, but it typically does not require the sophisticated hardware (e.g. stepping motors, linear actuators, etc.) related to mechanical methods which prevent these approaches to obtain the level of scalability and compactness that can be achieved with bacteria. Unlike electrical and magnetic methods, the method proposed here is also independent of the property of the micro-parts being manipulated such the dielectric characteristics.

\section{CONCLUSION}

Compared to self-assembly, controlled assembly is extremely versatile allowing the assembly of complex heterogeneous micro-structures. But a scaleable and flexible approach to controlled assembly where high levels of costeffective parallelisms can be achieved is required while being independent of the dielectric properties of the components being manipulated. Optical tweezers have been used extensively to manipulate micron-sized dielectric particles using trapping force in the typical range of 1-100 $\mathrm{pN}$. For larger force, a mechanical approach becomes necessary. In all cases, issues such as complexity, power and overall size of the each platform prevent them to be considered for cost-effective mass-scale micro-assembly processes. Here we show that micro-components can be manipulated accurately from the thrust force generated by flagellated Magnetotactic Bacteria (MTB) to assemble micro-structures. The force exerted on the components is not only independent of the dielectric property and scalable from a few $\mathrm{pN}$ with increments in the order of $4 \mathrm{pN}$ while being able to reach levels beyond the range of forces possible with optical tweezers, but the approach is highly scalable suggesting its potential for the implementation of costeffective mass-scale micro-assembly.

\section{ACKNOWLEDGMENT}

The authors acknowledge Maryline Thomas of the NanoRobotics Laboratory at EPM for making the building blocks used by the bacteria to build the Step pyramid.

\section{REFERENCES}

[1] S. Martel, Method and system for controlling micro-objects or microparticles, U.S. Pat. Appl. 11/145,007, 2005.

[2] S. Martel, "Controlled bacterial micro-actuation," Proc. Int. Conf. on Microtech. in Med. and Biol. (MMB), Okinawa, Japan, 2006.

[3] S. Martel, O. Felfoul, and M. Mohammadi, "Flagellated bacterial nanorobots for medical interventions in the human body," $2^{\text {nd }}$ IEEE RAS/EMBS Int. Conf. on Biomedical Robotics and Biomechatronics (BioRob), Scottsdale, AZ, USA, 2008.

[4] S. Martel, "Targeted delivery of therapeutic agents with controlled bacterial carriers in the human blood vessels," $2^{\text {nd }}$ ASM/IEEE EMBS Conf. on Bio, Micro and Nanosystems, San Francisco, USA, 2006.

[5] S. Martel, M. Mohammadi, O. Felfoul O., Z. Lu, and P. Pouponneau, "Flagellated magnetotactic bacteria as controlled MRI-trackable propulsion and steering systems for medical nanorobots operating in the human microvasculature," Int. Journal of Robotics Research (IJRR), 2009

[6] S. Martel, C. Tremblay, S. Ngakeng, and G. Langlois, "Controlled manipulation and actuation of micro-objects with magnetotactic bacteria," Appl. Phys. Lett., 89, pp. 233804-6, 2006.

[7] S. Martel S., W. André, M. Mohammadi, Z. Lu and O. Felfoul, "Towards Swarms of Communication-enabled and intelligent sensotaxis-based bacterial microrobots capable of collective tasks in an aqueous medium," IEEE Int. Conf. on Robotics and Automation (ICRA), 2009.

[8] H. Debarros,D. M. S. Esquivel, and M. Farina, "Magnetotaxis," Sci. Progr., 74, pp. 347-359, 1990.

[9] K.F. Böhringer, R.S. Fearing, and K.Y. Goldberg, "Microassembly," in Handbook of Industrial Robotics, S. Y. Nof, Ed., $2^{\text {nd }}$ ed. New York: Wiley, 1999.

[10] M. J. Kim, "Bacterial Flows: Mixing and Pumping in Microfluidic Systems Using Flagellated Bacteria," Ph.D. Thesis, Brown University, May 2005. 